\title{
Study on Leaf Functional Traits of Main Tree Species in Songshan Mountain
}

\author{
Yifu Yuan", An Mao", *, Yujie Luo ${ }^{2, *}$ \\ ${ }^{1}$ College of Forestry, Shandong Agricultural University, Taian, China \\ ${ }^{2}$ Tourism School, Shandong Women's University, Jinan, China
}

Email address:

yuanyf16@163.com (Yifu Yuan), dannymaoan@126.com (An Mao), yuaneco@sdau.edu.cn (Yujie Luo)

${ }^{*}$ Corresponding author

\section{To cite this article:}

Yifu Yuan, An Mao, Yujie Luo. Study on Leaf Functional Traits of Main Tree Species in Songshan Mountain. American Journal of Agriculture and Forestry. Vol. 8, No. 6, 2020, pp. 265-269. doi: 10.11648/j.ajaf.20200806.15

Received: November 1, 2020; Accepted: November 18, 2020; Published: November 27, 2020

\begin{abstract}
The functional traits of plant leaves are closely related to the habitat environment of plants, which are the most sensitive organs to environmental changes. Through the study of plant leaf traits, we can understand the growth status of plant species and their tolerance to adverse environmental conditions such as barren and drought. The mixed artificial communities of Cotinus coggygria and Platycladus orientalis were investigated by transect method in Songshan Mountain. We selected three native plants (Rhamnus parvifolia, Lespedeza bicolor and Vitex negundo) and a kind of introduced tree C. coggygria as research objects. There were significant differences in leaf functional traits among these four plants. The leaf area of $C$. coggygria was much larger than that of the other three plants, while the specific leaf area (SLA) of L. bicolor and R. parvifolia was significantly larger than that of C. coggygria, which was a typical trait strategy to adapt to low light environment. The SLA of C. coggygria was smaller than that of other plants, which was beneficial to improve water use efficiency. But the difference of SLA between C. coggygria and other plants did not reach a significant level, which indicated that there were similarities in drought adaptability between the native plants and the introduced plants.
\end{abstract}

Keywords: Leaf Functional Traits, Trait Strategy, Tree Species, Introduced Plants

\section{Introduction}

Water is an essential resource in plant growth, which plays an important role in the whole life cycle of plants. As a main resource or medium, water participates in the metabolic processes of photosynthesis, nutrient transport and transpiration. The acquisition, maintenance and utilization of water by plants are of great significance for individual survival, interspecific competition and community formation. In order to adapt to different climate and water conditions, plants have evolved different adaptive strategies, such as isochoric and non-isochoric regulation behaviors [1, 2]. Through the combination of a series of related traits, the survival and distribution of plants can be realized [3].

With the increasing global climate change, the original spatial and temporal pattern of precipitation has been changed, and extreme meteorological events have occurred frequently, resulting in huge losses of agricultural and forestry production. Frequent meteorological drought causes changes in water conditions of plant communities $[4,5]$. Drought stress restricts the survival and growth of plants, reduces plant productivity, and has a profound impact on the species composition and structure of plant communities [6,7]. In the process of vegetation restoration, the introduction of woody plants is restricted by site conditions, especially soil moisture, which has a great impact on the survival rate and growth of seedlings.

Due to the long-term overdevelopment (agricultural planting, grazing and woodcutting) in the history of low mountains and hills in Shandong Province, the original vegetation has been completely destroyed, the surface soil erosion is serious, resulting in shallow soil layer, low soil water and fertilizer conservation capacity[7]. There is a large area of arid barren mountains in Shandong Province, which need to be afforested. According to statistics, Shandong Province has a total area of 157000 square kilometers, of which mountains and hills account for nearly $40 \%$ of the total 
area, mainly including the low mountains and hills in the middle and south of Shandong Province and the hills in Jiaodong. After the founding of the people's Republic of China, Shandong Province has made great achievements in forestry construction. The forest area has reached 33629 square kilometers, and the forest coverage rate has reached $21.42 \%$ (up to 2008). It is mainly concentrated in areas with good site conditions such as plains and some hilly areas [8]. However, the overall forest coverage rate is relatively low in mountainous and hilly areas, especially in the mountainous and hilly areas with shallow soil layer.

In recent years, relying on the support of various projects and funds, Shandong Province vigorously promotes afforestation and vegetation restoration. There are large areas of new afforestation every year. There are a lot of difficult site conditions, many bare rocks, thin soil layer, poor water supply and other site factors. The survival rate of afforestation is low. It is particularly important to protect and improve the survival rate of seedlings, which is directly related to the effect of afforestation.

Plant functional traits are the structural and physiological characteristics of plants that respond to environmental changes and have a certain impact on ecosystem functions [9, 10]. Different from the traditional plant characters, only describe the individual plant, plant functional traits more emphasize the role of plant adaptive environment and impact on the environment, which has stronger practical significance in the research. Therefore, plant functional traits provide a good perspective for studying the relationship between plant and environment.

In recent years, the study of plant functional traits has become a hot spot in the study of plant from individual, community to ecosystem. In 2004, 33 scientists from the United States, Australia and South Africa jointly published a research paper on global leaf economic spectrum [11]. Based on the study of 2548 plant species from 175 sites around the world, six main leaf functional traits, such as specific leaf weight, net photosynthetic rate, respiration rate, leaf nitrogen content, leaf phosphorus content and leaf life span, were selected to reveal the relationship between plant leaf functional traits and environmental factors at the global scale for the first time through the correlation study with major climate factors. The results showed that there was a close relationship between plant functional traits and climate at regional scale, and plants at both ends of leaf economic spectrum showed different adaptive strategies. In the following research, scientists have carried out fruitful work on plant functional traits. Reich (2014) expanded the original leaf economic spectrum to a universal "fast slow" plant economic spectrum hypothesis, including leaf stem root and carbon nutrient water, and proposed that the dominant factor driving the biomass allocation pattern of forest plants such as leaves, stems and roots is temperature at the global scale [12]. The results of meta-analysis showed that the leaf morphological characters of more than 800 plants in northern China were significantly affected by water gradient and temperature in growing season [13]. Specific leaf area and leaf width of 386 perennial plants in Australia increased significantly with the increase of soil moisture [14]. The leaf area and specific leaf area of Salsola nitraria increased significantly with the increase of soil moisture, but the growth of plants was inhibited under poor water conditions [15].

Through the study of plant functional traits, the growth status of plants can be reflected. At the same time, the comparison between different plants has certain guiding significance for screening afforestation plants and formulating differential and accurate forest management measures. Based on this, we selected a sample plot of artificial vegetation restoration and selected three native plants (Rhamnus parvifolia, Lespedeza bicolor and Vitex negundo) and a kind of introduced tree Cotinus coggygria as research objects.

In the background of vegetation restoration, based on the comparison of the main leaf functional traits of these four plants, we put forward the following two scientific questions:

1 Are there any differences in the main leaf functional traits between introduced plant (C. coggygria) and native plants (R. parvifolia, L. bicolor and V. negundo)?

2 Does the difference of main leaf functional traits affect plant tolerance to drought?

\section{Methods}

\subsection{Study Area}

Songshan Mountain in Linqu, Weifang City, with an altitude of 760 meters, is the second largest mountain in Linqu. It is located at the edge of the middle mountain area of Shandong Province, which is a crisscross zone of low mountains, hills and plains. The climate is temperate monsoon continental climate, with four distinct seasons, with simultaneous rain and heat, and obvious dry and wet seasons. The annual average precipitation is about $700 \mathrm{~mm}$, the average temperature is $12.8^{\circ} \mathrm{C}$, the annual average sunshine is about $2514 \mathrm{~h}$, and the frost free period is $203 \mathrm{~d}$. The existing vegetation is mainly the common mesophytic and xerophilic shrubs and herbs in North China, as well as Platycladus orientalis, Robinia pseudoacacia and other industrial forests.

\subsection{Field Investigation and Sample Collection}

This study was conducted in Songshan. The community of mixed plantation of C. coggygria and Platycladus orientalis was investigated by transect survey. Each sample line is $30 \mathrm{~m}$ long and $10 \mathrm{~m}$ wide. In each transect, 4 species of trees and shrubs including C. coggygria, V. negundo, R. parvifolia and $L$. bicolor were sampled. Five mature and well growing individuals were selected for each sample line, and the fully expanded leaves without diseases and insect pests were collected. Leaves on the outer branches of the crown were selected. Put the leaves were put put into bags, between two pieces of wet filter paper, taken back to the laboratory, and put in the refrigerator fresh-keeping layer for storage.

\subsection{Measurement of Leaf Morphological Characters}

Epson perfection v700 Photo Scanner was used to scan the 
leaves, and winfolia pro2009a (Regent instruments, Inc., Quebec, Canada) was used to analyze and calculate leaf area, leaf circumference and other traits. The dry weight of leaves was obtained by drying constant weight method, and was dried in the oven at $60{ }^{\circ} \mathrm{C}$ for 48 hours to constant weight. Then the specific leaf area (SLA) was calculated by using the obtained leaf area size and leaf dry weight:

$$
\mathrm{SLA}=\text { leaf area }\left(\mathrm{cm}^{2}\right) / \text { leaf dry weight }(\mathrm{g})
$$

\subsection{Data Processing}

After the experiment, the measured data were processed in Excel, and the mean value, standard deviation, significance analysis (95\% confidence interval) and one way ANOVA analysis of variance were calculated by SPSS 17.0 software (SPSS Inc., Chicago, USA), and then the related results were plotted by Origin8.0 (Originlab Co., Northampton, MA).

\section{Results}

\subsection{Leaf Traits of Main Woody Plants in Songshan Mountain}

At the end of the experiment, we analyzed the main leaf functional traits of these four plants by one-way ANOVA. It
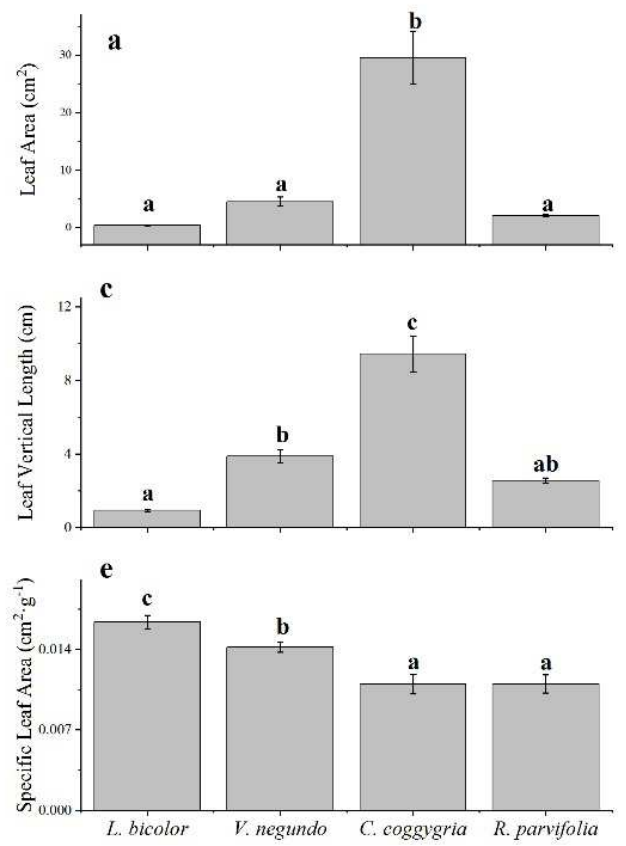

can be seen that there are significant differences in the main leaf functional traits of the four plants, indicating that although these plants are distributed in the same habitat, there are great differences in leaf morphology and specific leaf area.

Table 1. Results of one-way ANOVA (F-values and significance levels) for leaf functional traits of different plants.

\begin{tabular}{lll}
\hline Traits & $\mathbf{F}$ & $\boldsymbol{p}$ \\
\hline Leaf area & 28.24 & $0^{* * *}$ \\
leaf perimeter & 50.65 & $0^{* * *}$ \\
Leaf vertical length & 38.52 & $0^{* * *}$ \\
Leaf avghorizontal width & 84.27 & $0^{* * *}$ \\
Leaf aspect ration & 22.03 & $0^{* * *}$ \\
Specific leaf area & 11.96 & $0^{* * *}$ \\
\hline
\end{tabular}

Significant effects are indicated by asterisks: $* * * p<0.001, * * p<0.01$ and $* p$ $\leq 0.05$.

It can be seen that there are significant differences in the SLA of the four plants (Figure 1). L. bicolor has the largest specific leaf area, which is $0.016 \mathrm{~m}^{2} \cdot \mathrm{g}^{-1}$, followed by $V$. negundo, which is $0.014 \mathrm{~m}^{2} \cdot \mathrm{g}^{-1}$. The SLA of $R$. parvifolia and $C$. coggygria is significantly smaller than the above two species, both $0.011 \mathrm{~m}^{2} \cdot \mathrm{g}^{-1}$. Leaflet area of $C$. coggygria is much larger than the other three plants, up to $29.57 \mathrm{~cm}^{2}$; while $L$. bicolor is the lowest, only $0.40 \mathrm{~cm}^{2} ; V$. negundo and $R$. parvifolia are $4.54 \mathrm{~cm}^{2}$ and $2.08 \mathrm{~cm}^{2}$, respectively.
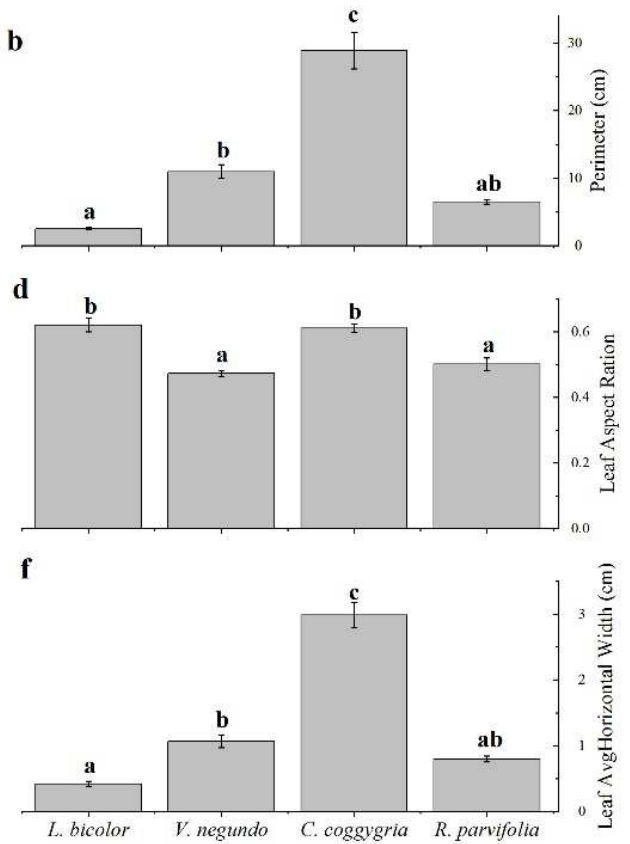

Figure 1. Leaf functional traits of different plants. Values are presented as mean \pm SE. Different letters denote significant differences at $p<0.05$ by Duncan `s test.

We selected the vertical distance of the main leaf vein direction as the leaflet height. It can be seen that C. coggygria has the largest leaflet height of $9.45 \mathrm{~cm}$, which is significantly larger than that of the other three plants; Lespedeza bicolor has the lowest leaflet height, which is $0.93 \mathrm{~cm}$; the leaflet height of $V$. negundo and $R$. parvifolia are $3.88 \mathrm{~cm}$ and $2.55 \mathrm{~cm}$ respectively. We also calculated the average width of leaves. Through comparison, we can see that $C$. coggygria has the largest leaflet width of $2.99 \mathrm{~cm}$, which is significantly larger than the other three plants; L. bicolor has the smallest, which is $0.42 \mathrm{~cm} ; V$. negundo and $R$. parvifolia are $1.07 \mathrm{~cm}$ and $0.80 \mathrm{~cm}$ respectively. There are significant differences in leaf width height ratio of these four plants. The leaf width height ratio of Lespedeza bicolor (0.62) and C. coggygria (0.61) is significantly higher than that of $V$. negundo $(0.47)$ and $R$. parvifolia (0.50). The leaves of $V$. negundo and $R$. parvifolia 
are longer and narrower than those of L. bicolor and $C$. coggygria.

The leaf perimeter of different plants in Songshan Mountain is similar to the results of leaf area. Among the four plants, $C$. coggygria has the largest leaf perimeter of $28.86 \mathrm{~cm}$, which is significantly larger than that of the other three native plants, while L. bicolor is $2.53 \mathrm{~cm}, V$. negundo and R. parvifolia are $10.99 \mathrm{~cm}$ and $6.49 \mathrm{~cm}$ respectively.

\subsection{Comparison of Leaf Traits Between Native Plants and Introduced Plants in Songshan Mountain}

According to the previous analysis, there are significant differences in leaf function among the four plants. However, $R$. parvifolia, L. bicolor and $V$. negundo are the original plants in the sample plot, while $C$. coggygria is the planting plants in the artificial vegetation restoration, so we compare the two kinds of plants according to the source of plant species. Considering the obvious differences in leaf area and perimeter in leaf morphological traits, we selected SLA for comparison (Figure 2).

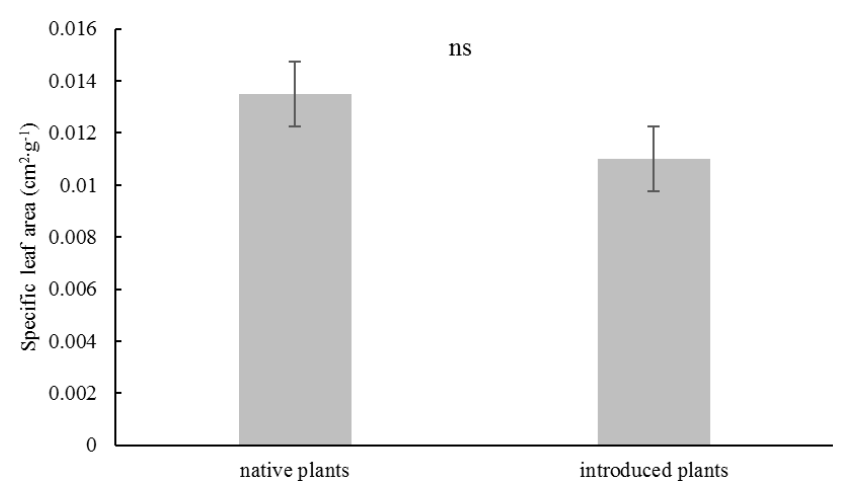

Figure 2. Independent sample t test of SLA of native and introduced plants Values are presented as mean $\pm S E$. Different letters denote significant differences at $p<0.05$ by Duncan`s test.

It can be seen that the SLA of $C$. coggygria is smaller than that of native plants, but the difference is not significant. Although there are obvious differences in leaf morphology, the SLA reflects a similar type of leaf carbon input and distribution.

\section{Discussion}

\subsection{Comparison of Leaf Functional Traits of Different Plant Species in Songshan Mountain}

In this study, there were significant differences in leaf functional traits among four plants in the same community. As an important organ for plants to receive light and carry out photosynthesis, leaf directly determines its ability to obtain light. Generally, the larger the leaf area of a plant species, the greater the area of photosynthesis it can carry out. Therefore, there is a close relationship between the leaf area of a plant species and its yield $[10,13]$. In this study, the leaf area of the four plants was very different. The leaf area of C. coggygria was much larger than that of the other three plants, while that of L. bicolor and R. parvifolia was smaller. As a small tree, $C$. coggygria has higher plant height than $L$. bicolor and $R$. parvifolia. Therefore, C. coggygria occupies the top of the canopy in the community and has good light acquisition conditions. Lespedeza and $R$. parvifolia are located at the bottom of the canopy, and their leaf area is small, but the number of leaves is large, and they also have certain light acquisition ability.

In addition, the results showed that the specific leaf area of different plants varied greatly. The SLA reflects the carbon allocation strategy of plants in leaf construction. The larger SLA, the larger the leaf area produced by carbon input per unit mass, which is of great significance for light acquisition [16, 17]. The difference of SLA between plant species also represents different survival strategies. The specific leaf area of L. bicolor and $R$. parvifolia at the bottom of canopy was significantly larger than that of $C$. coggygria, which was also a typical trait strategy to adapt to low light environment. At the same time, we also found that the larger the leaf area, the smaller the ratio of length to width. Plants occupy different niches through functional traits, and utilize environmental resources such as sunlight, water and mineral nutrition at different levels. In order to reduce interspecific competition and facilitate the survival of species in the community, plants can achieve niche differentiation through the difference of characters.

\subsection{Response of Leaf Traits of Different Plant Species in Songshan Mountain to Drought Stress}

In general, plants reduce transpiration by reducing leaf area to adapt to physiological drought. The leaf area of the three native shrubs in this study is small, which is consistent with the general research conclusion [18-21]. As a small tree, $C$. coggygria has larger leaf area than the three shrubs. But compared with other local trees, its leaf area is relatively small, and it has better adaptability to drought. The SLA can reflect the utilization of organic matter and nutrition and the strategy of adaptation to the environment. The SLA of plants under drought condition is small. In this study, the SLA of $C$. coggygria was smaller than that of other plants, which indicated that it invested more in unit area, which was beneficial to improve water use efficiency. Plants with small SLA have stronger resistance to herbivorous natural enemies, wind and frost disasters. In addition, plants with small SLA have longer leaf life, which helps to prolong leaf carbon accumulation and improve resource utilization efficiency. It should be noted that the difference of SLA between $C$. coggygria and other plants did not reach a significant level, which indicated that there were similarities in drought adaptability between the native plants and the introduced plants.

\section{Conclusion}

In this study, Rhamnus parvifolia, Lespedeza bicolor, Vitex negundo, and C. coggygria were selected as research objects. The results showed that there were significant differences in 
leaf functional traits among these four plants. The leaf area of $C$. coggygria was much larger than those of the other three plants, while the SLAs of L. bicolor and R. parvifolia were significantly larger than that of $C$. coggygria, which was a typical trait strategy to adapt to low light environment. The SLA of C. coggygria was smaller than those of the other three plants, which was beneficial for the improvement of water use efficiency. However, the difference of SLA between $C$. coggygria and other plants did not reach a significant level, indicating that there were similarities in drought adaptability between the native plants and the introduced plants.

\section{Author Contributions}

The Manuscript was written through contributions of all authors. All authors have given approval to the final version of the manuscript.

\section{Conflicts of Interest}

The authors declare that they have no competing interests.

\section{Acknowledgements}

The research was supported by National Science Foundation of China (Project No. 31500464).

\section{References}

[1] Zhong, Q. L., Liu, L. B., Xu, X., Yang, Y., Guo, Y. M., Xu, H. Y., Cai, X. L. and Ni, J. (2018). Variations of plant functional traits and adaptive strategy of woody species in a karst forest of central Guizhou Province, southwestern China. Chinese Journal Plant Ecology, 42(5): 562-572.

[2] Chen, W., Wang, J. H., Ma, R. J., Qi, W., Liu K, Zhang, L. N. and Chen, X. L. (2016). Variance in leaf functional traits of 89 species from the eastern Guangdong of China. Chinese Journal of Ecology, 35: 2101-2109.

[3] Craine, J. M., Lee, W. G., Bond, W. J., Williams, R. J. and Johnson, L. C. (2005). Environmental constraints on a global relationship among leaf and root traits of grasses. Ecology, 86: 12-19.

[4] Chen, X. (2000). The relationship of trees phenology growth season and temperature growth seasons: an example of Taunus mountains in the middle of German. Acta Meteorology Sinica, 58:721 737.

[5] Kramer, K., Friend, A. D. and Leinonen, I. (1996). Modelling comparison to evaluate the importance of phenology for the effects of climate change in growth of mixed temperature-zone deciduous forests. Climate Research, 7: 31 41.

[6] Guo, K., Liu, C. C. and Dong, M. (2011). Ecological adaptation of plants and control of rocky-desertification on karst region of Southwest China. Chinese Journal of Plant Ecology, 35: 991-999.

[7] Liu, H. W., Liu, W. D., Wang, W., Chai, J. and Tao, J. P. (2015). Leaf traits and nutrient resorption of major woody species in the karst limestone area of Chongqing. Acta Ecologica Sinica, 35: 4071-4080.

[8] Zhao, Q. K. (2000). Ecological agriculture of weak ecological areas in mountain area of central south of Shandong Province --A case study in Yimeng Mountain area. Journal of Mountain Science, 3: 253-257.

[9] Shao, S. X., Dong, Z., L, H. li., Xu, T. T., Zhang, Q. J. and Zhu, C. (2015). Herbaceous Community Characteristics and Species Diversity of Young Artificial Forest of Degraded Granite Hilly Areas in Central-south of Shandong Province. Forest Resources Management, 1: 77-83.

[10] Figueroa, J. A. and Armesto, J. J. (2001). Community-wide germination strategies in a temperate rainforest of Southern Chile: Ecological and evolutionary correlates. Australian Journal of Botany, 49: 411-425.

[11] Auger, S. and Shipley, B. (2013). Inter-specific and intra-specific trait variation along short environmental gradients in an old-growth temperate forest. Journal of Vegetation Science, 24: 419-428.

[12] Wright, I. J., Reich, P. B., Westoby, M., et al. (2004). World-wide leaf economics spectrum. Nature, 428(6985): 821-827.

[13] Reich, P. B. (2014). The world-wide 'fast-slow' plant economics spectrum: a traits manifesto. Journal of Ecology, 102(2): 275-301.

[14] Meng, T. T., Ni, J. and Harrison, S. P. (2009). Plant morphometric traits and climate gradients in northern china: a meta-analysis using quadrat and flora data. Annals of Botany, 104(6): 1217-1229.

[15] Fonseca, C. R., Overton, J. M., Collins, B. and Westoby, M. (2001). Shift in trait-combination along rainfall and phosphorus gradients. Journal of Ecology, 88(6): 964-977.

[16] Zhao, X. F., Xu, H. L. and Zhang, P. (2014). Influence of nutrient and water additions on functional traits of Salsola nitraria in desert grassland. Chinese Journal Plant Ecology, 38(2): 134-146.

[17] Poorter, H., Niinemets, U. and Poorter L. (2009). Causes and consequences of variation in leaf mass per area (LMA): a meta analysis. New Phytologist, 182: 565-588.

[18] Yao, T. T., Meng, T. T., Ni, J., Yan, S., Feng, X. H. and Wang, G. H. (2010). Leaf functional trait variation and its relationship with plant phylogenic background and the climate in Xinjiang Junggar Basin, NW China. Biodiversity Science, 18(2): 188-197.

[19] Villar, R., Merino, J. (2001). Comparison of leaf construction costs in woody species with differing leaf life spans in contrasting ecosystems. New Phytologist, 151: 213-226.

[20] Cunningham, S. A., Summerhayes, B. and Westoby, M. (1999). Evolutionary divergences in leaf structure and chemistry, comparing rainfall and soil nutrient gradients. Ecological Monographs, 69: 569-588.

[21] Dong, T. F., Feng, Y. L., Lei, Y. B. and Zhang, L. K. (2012). Comparison on leaf functional traits of main dominant woody species in wet and dry habitats. Chinese Journal of Ecology, 31(5): 1043-1049. 